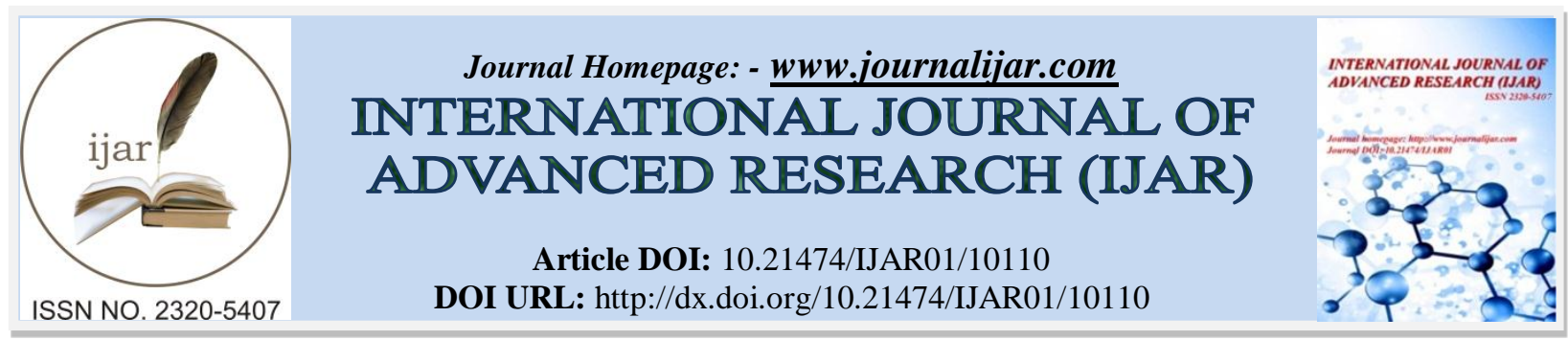

RESEARCH ARTICLE

\title{
SYNTHESIS AND EVALUATION OF A MUCO-ADHESIVE HYDROGEL CONTAINING DOXYCYCLINE AND METRONIDAZOLE FOR LOCAL DRUG DELIVERY FOR THE TREATMENT OF PERIODONTITIS.
}

Dr. Shashi Kiran S and Dr. Narayan N Valavalkar.

Department of Periodontics, College of Dental Sciences, Davangere, Karnataka, India.

\section{Manuscript Info}

Manuscript History

Received: 14 September 2019

Final Accepted: 16 October 2019

Published: November 2019

Key words:-

Doxycycline, Metronidazole, Local drug delivery, Hydrogel, Periodontitis.

\section{Abstract}

Background: The current knowledge and understanding of Periodontitis shows that the best chance for clinical improvement may be obtained from implementing complementary strategies like the reduction of the bacterial burden, host modulation, and risk factor modification that targets different aspects of the periodontal balance. The reduction of bacterial burden by Scaling and root planing is the cornerstone of treatment and can be augmented by the use of local antimicrobials with or without host modulation. The aim of the present study was to synthesize and evaluate a muco-adhesive hydrogel matrix carrying 1\% Metronidazole (a local antimicrobial) and 2\% Doxycycline (a host modulating agent) in the treatment of Periodontitis.

Methodology: A chitosan based hydrogel containing 1\% metronidazole and 2\% doxycycline was synthesized and evaluated for Minimum Inhibitory Concentration (MIC). Twenty Periodontitis patients with at least one site of probing pocket depth $5-7 \mathrm{~mm}$ were selected and randomly divided in to control and test groups. Full mouth Scaling and root planning was done in all 20 patients at the first visit and the baseline clinical parameters were recorded. Only the test group patients received intra-pocket delivery of the hydrogel containing $1 \%$ metronidazole and 2\% doxycycline. All Patients were recalled after 14 days and 30 days for assessment of clinical parameters.

Results: The Minimum Inhibitory Concentration of the prepared hydrogel against the prime periodontal pathogens, Porphyromonas gingivalis, Tanerella forsythia, Fusobacterium nucleatum, Prevotella intermedia was found to be as low as $0.4 \mu \mathrm{g} / \mathrm{ml}$. Both groups showed a statistically significant decrease in gingival index scores. However, test group patients showed a significantly higher reduction in probing pocket depths and gingival index scores when compared to the control group. There was no significant improvements in clinical attachment levels in both groups.

Conclusion: In this study, the prepared hydrogel containing doxycycline and metronidazole showed the potential to be used as a local drug delivery agent as an adjunct to scaling and root planing in the treatment of Periodontitis. However, future studies with larger sample size and longer duration are needed to explore the efficacy of the gel. 


\section{Introduction:-}

Periodontitis is a chronic multifactorial inflammatory disease associated with dysbiotic plaque biofilms and characterized by progressive destruction of the tooth-supporting apparatus (Papapanou et al., 2018). It is characterized by microbially-associated, host-mediated inflammation that results in loss of periodontal attachment. The pathophysiology of the disease has been characterized in its key molecular pathways, and ultimately leads to activation of host-derived proteinases that enable loss of marginal periodontal ligament fibers, apical migration of the junctional epithelium, and allows apical spread of the bacterial biofilm along the root surface (Tonetti et al., 2018).

The approach to treat such a complex multifactorial disease should involve additional complimentary treatment strategies. The reduction in bacterial burden by scaling and root planning (SRP) is the cornerstone of treatment. Scaling and root planning is a mechanical therapy which aims at removing all the soft and hard deposits off the tooth surface to achieve an overall decrease in microbial load in the local environment. But local mechanical debridement has been shown to have certain drawbacks like inability to eliminate pathogens from isolated niches like deep periodontal pockets and furcation areas where mechanical instrumentation is extremely difficult. Hence the adjunctive use of antimicrobial agents in treating Periodontitis might effectively reduce or eliminate pathogenic organisms from root furcation areas, deep periodontal pockets and soft tissue wall of the pockets. However, systemically administered antibiotics have several disadvantages like large doses, first pass metabolism leading to lower concentrations in gingival crevicular fluid, systemic side effects, risk of developing drug resistance and a lower patient compliance (Jepsen S et al., 2011). Hence delivering the antimicrobial agent directly into the local pocket environment is a more logical approach than systemic administration.

Since most of the destruction that happens in Periodontitis is due to an exaggerated and dysregulated immunoinflammatory response of the host and certain host-derived pro-inflammatory cytokines, Matrix-metalloprotienases (MMPs) and prostaglandins, it might be plausible to regulate the host's response towards microbial plaque. Even though the intent of the release of these inflammatory mediators are protective in nature, they paradoxically cause extensive collateral damage to the surrounding tissues giving rise to the characteristic clinical signs of the disease. The concept of 'Host modulation' aims at regulating and balancing the body's immune response towards the microbial biofilm in order to limit the excessive destruction of the vital periodontal structures (Preshaw PM, 2008). Hence the treatment of Periodontitis with local mechanical debridement can be augmented by the use of antimicrobials agents and host modulation.

Metronidazole, a nitro-imidazole compound is a front line bactericidal chemotherapeutic agent for treating infections by anaerobic bacteria (Löfmark et al., 2010). It is effective against most of the periodontal pathogens including Porphyromonas gingivalis and Prevotella intermedia. Hence Metronidazole can be a potent antimicrobial agent in the treatment of Periodontitis. Doxycycline belongs to the tetracycline family of drugs. It is a broad spectrum semisynthetic bacteriostatic antibiotic with a dual mechanism of action. It inhibits the protein synthesis and suppresses the growth of microorganisms and also inhibits the host derived pro-inflammatory cytokines, MMPs and prostaglandins indicating its potential as a host modulating agent (Golub et al. 1998, Golub et al. 1991, Golub et al. 1992).

In the current study, an attempt was made to combine two different treatment strategies, local antimicrobial therapy and host modulation to augment the primary strategy of mechanical debridement. The aim was to synthesize and evaluate a muco-adhesive hydrogel matrix carrying metronidazole (an anti-microbial) and doxycycline (an antimicrobial and host modulating agent) for local drug delivery in the treatment of Periodontitis.

\section{Materials and Methods:-}

The study was approved by the author's institutional review board. The study was divided into three phases: Pharmacological, Microbiological and Clinical phase.

\section{Pharmacological phase:}

A 5\% Carboxy-methyl Chitosan (CMCS) hydrogel was prepared which contains $1 \%$ Metronidazole and $2 \%$ Doxycycline. 
Preparation of gel: $1250 \mathrm{mg}$ of CMCS was dissolved in $25 \mathrm{ml}$ of distilled water to obtain a $5 \%$ solution. $0.25 \mathrm{~g}$ of menthol (permeability enhancer), 200mg of analytical grade Metronidazole and 500mg of Doxycycline hyclate was added to the solution and stirred. $1 \mathrm{ml}$ of glycerine was added as a plasticizer. Two drops of Tri-ethanolamine was added to adjust the $\mathrm{P}_{\mathrm{H}}$ to 6 . The solution was kept under sterile conditions, overnight for gel formation. The resultant mixture was a golden yellow coloured, viscous, flowable formulation (Fig. 1). Each millilitre of hydrogel would contain 10mg of Metronidazole and 20mg of Doxycycline.

\section{Microbiological phase:}

Minimum inhibitory concentration (MIC): The Minimum inhibitory concentration of the prepared hydrogel was tested against the prime periodontal pathogens, Porphyromonas gingivalis, Tanerella forsythia, Fusobacterium nucleatum, Prevotella intermedia.

Procedure: Nine dilutions of each drug have to be done with Thioglycollate broth for MIC. In the initial tube, 20 microliter of drug was added into 380 microliter of Thioglycollate broth. For dilutions, 200 microliter of Thioglycollate broth was added into the next nine tubes separately. Then from the initial tube, 200 microliter was transferred to the first tube containing 200 microliter of Thioglycollate broth. This was considered as 10-1 dilution. From 10-1 diluted tube 200 microliter was transferred to second tube to make 10-2 dilution. The serial dilution was repeated up to 10-9 dilution for each drug. From the maintained stock cultures of required organisms, 5 microliter was taken and added into $2 \mathrm{ml}$ of Thioglycollate broth. In each serially diluted tube, 200 microliter of above culture suspension was added. The tubes were incubated for $48-72$ hours in anaerobic jar at $37^{\circ} \mathrm{C}$ and observed for turbidity

The MIC of the prepared hydrogel against the prime periodontal pathogens, Porphyromonas gingivalis, Tanerella

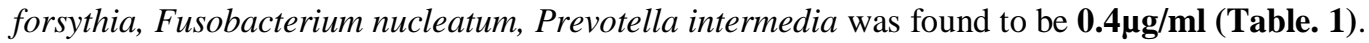

\section{Clinical phase:}

Twenty patients from Outpatient Department of Periodontics, College of Dental Sciences, Davangere, Karnataka, India, with at least one periodontal pocket of depth $5-7 \mathrm{~mm}$ (Fig. 2) were selected and divided randomly into test and control groups using the coin toss method. A written informed consent was obtained from all patients.

\section{Group 1 (control): SRP only}

Group 2 (test): SRP + intra-pocket placement of hydrogel.

Pregnant patients, smokers, patients with any systemic diseases, patients with a previous history of treatment of Periodontitis, patients who had taken antibiotics within three months before the study period or patients with history of use of antiseptic mouth rinses were excluded. All patients underwent full mouth SRP at the first visit and baseline clinical parameters, probing pocket depth (PPD), clinical attachment loss (CAL), and Gingival index (GI) were recorded before SRP.

Only patients in the test group received intra-pocket placement of the hydrogel. Approximately $0.5 \mathrm{ml}$ of gel was placed in the periodontal pocket around the tooth using a syringe with a wide-bore blunt tip (Fig. 3a and Fig. 3b) and a periodontal pack was placed. After the treatment, patients were instructed to avoid touching the treated areas, eating hard, crunchy or sticky foods for one week, and to postpone brushing for a period of 12 hours. All the patients were recalled after 14 days and 30 days for re-evaluation of the clinical parameters. The periodontal pack was removed at the $14^{\text {th }}$ day.

\section{Results:-}

All the required data were obtained and sent for statistical analyses using SPSS version 18.0 IBM SPSS (IBM Inc. Chicago). Statistical analysis was done using ANOVA and Students $t$ test for parametric data and Friedman test and Mann-Whitney test for non-parametric data. A P value $<0.05$ was considered statistically significant.

\section{Intragroup analysis: \\ Control group:}

The mean PPD at baseline, 14days and 1 month in control group was $5.9 \pm 0.73 \mathrm{~mm}, 5.3 \pm 0.94 \mathrm{~mm}$ and $5.3 \pm 0.67 \mathrm{~mm}$ respectively. There was no statistically significant difference in PPD between baseline, $14^{\text {th }}$ and $30^{\text {th }}$ days. The mean CAL at baseline, $14^{\text {th }}$ and $30^{\text {th }}$ day was $5.2 \pm 1.1 \mathrm{~mm}, 4.9 \pm 0.99 \mathrm{~mm}, 4.9 \pm 1.1 \mathrm{~mm}$ respectively. There was no statistically significant difference in CAL between baseline, $14^{\text {th }}$ and $30^{\text {th }}$ days. There was statistically significant difference in mean gingival index score at baseline and 30 days ( $2.2 \pm 0.42$ and $1.0 \pm 0.47$ respectively) (Table. 2 ) 


\section{Test group:}

The mean PPD at baseline, $14^{\text {th }}$ and $30^{\text {th }}$ day in test group was $5.7 \pm 0.67 \mathrm{~mm}, 4.5 \pm 0.84 \mathrm{~mm}, 4.0 \pm 0.94 \mathrm{~mm}$. There was statistically significant difference in PPD between baseline and $14^{\text {th }}$ day $(\mathrm{p}=0.009)$. There was highly significant difference in PPD between baseline and $30^{\text {th }}$ day $(\mathrm{p}=0.00)$. The mean CAL at baseline, $14^{\text {th }}$ and $30^{\text {th }}$ days were $5.0 \pm 0.81 \mathrm{~mm}, 4.6 \pm 0.84 \mathrm{~mm}$ and $4.4 \pm 0.84 \mathrm{~mm}$ respectively. There was no statistically significant difference in CAL between baseline, $14^{\text {th }}$ and $30^{\text {th }}$ days. There was statistically significant difference in mean gingival index score at baseline and 30 days (2.2 \pm 0.42 and $0.3 \pm 0.48$ respectively) (Table. 3$)$.

\section{Intergroup analysis:}

Intergroup comparison revealed significantly higher reduction in mean PPD in the test group at 30 days $(\mathrm{p}=0.002)$. There was significantly higher reduction in mean gingival index scores in the test group at 14 and 30 days $(\mathrm{p}=0.005)$. There was no significant difference in mean clinical attachment levels between the 2 groups at 14 and 30 days (Table. 4$)$.

\section{Discussion:-}

The primary aim of this study was to prepare a muco-adhesive local drug delivery agent which has both antimicrobial and host modulation properties for the treatment of Periodontitis. Novak et al, in 2008 attempted to combine host modulation and local topical anti-microbial therapy as an adjunct to scaling and root planing using systemically administered Doxycycline 20mg (Periostat) and locally delivered Doxycycline gel. They concluded that combination therapy, including SRP, Host modulation, and Topical Anti-microbial therapy, provided significantly greater clinical benefits than SRP alone in the treatment of moderate to severe Chronic Periodontitis (Novak et al., 2008).

Carboxy-methyl chitosan (CMCS) is a water soluble, biocompatible and biodegradable polymer which is obtained from the reaction of chitosan with mono-chloroacetic acid and in alkaline condition (Chen SC et al., 2004). Due to its antimicrobial activity, film-forming ability, muco-adhesive property and solubility in wide range of $\mathrm{pH}$, it is used in medical and pharmaceutical areas mainly for the controlled release of drug (FR Abreu et al., 2009). Hence CMCS was chosen as the muco-adhesive polymer for the preparation of the hydrogel.

Metronidazole, a bactericidal, synthetic nitro-imidazole compound was chosen as the primary antimicrobial agent for its action against gram negative anaerobic periodontal pathogens. There is a generally accepted notion that the anti-microbial efficacy of a bacteriostatic antibiotic like doxycycline is compromised when combined with a bactericidal agent like metronidazole (Winkelhoff et al., 1996). However, in this study, Doxycycline was chosen for its property of inhibiting collagenase and pro-inflammatory cytokines for host modulation.

The concentrations of metronidazole $(1 \%$ or $10 \mathrm{mg} / \mathrm{ml})$ and doxycycline $(2 \%$ or $20 \mathrm{mg} / \mathrm{ml})$ were selected on the basis of previously published literature on MIC of $0.1-8 \mu \mathrm{g} / \mathrm{ml}$ and $0.25-6.0 \mu \mathrm{g} / \mathrm{ml}$, respectively (Larsen, 2002) (Sedlacek et al, 2007). In the current study, the concentration of drugs incorporated in the gel was greater than the minimum required (approximately 1600 and 2500 times greater for metronidazole and doxycycline respectively). This higher concentration of the drugs in the gel is to compensate for the potential loss of gel caused by the flow of gingival crevicular fluid and saliva. It also satisfies the requirement of a higher concentration of an anti-microbial to be effective against bacteria arranged in bio-films (antibiotic strength that is 500 times greater than the usual therapeutic dose may be needed to be effective against bacteria that have become arranged in bio-films (Greenstein, 2005)).

In the current study, the prepared muco-adhesive hydrogel was translucent, golden yellow in colour and had optimal viscosity which could potentially enhance its retention in the periodontal pocket. The gel was shown to have an extremely low minimum inhibitory concentration against periodontal pathogens, Porphyromonas gingivalis, Tanerella forsythia, Fusobacterium nucleatum, Prevotella intermedia. All four organisms were inhibited at a concentration as low as $0.4 \mu \mathrm{g} / \mathrm{ml}$ (Table. 1). Throughout the study, the gel was refrigerated at $4^{0} \mathrm{Celsius}$. Approximately $0.5 \mathrm{ml}$ of gel was placed in the periodontal pocket. $0.5 \mathrm{ml}$ of gel would contain $5 \mathrm{mg}$ of Metronidazole and 10mg of Doxycycline which is well beyond the MIC of gel.

Nastri et al in 2019, developed a controlled-release material containing Metronidazole (2\%) and Doxycycline (1\%) for the treatment of Periodontitis. They confirmed the in vitro efficacy of the newly formulated gel, both on planktonic species and on bacterial biofilm over a period of 13 days. Their controlled-release gel had an optimal 
final viscosity and muco-adhesive properties. They concluded that its employment could be useful for the treatment of periodontal and peri-implant diseases, where conventional therapy seems not successful (Nastri et al, 2019). Gad et al in 2017, developed a solid lipid micro particle encapsulating 5\% w/w doxycycline hydrochloride and 20\% w/w metronidazole for the treatment of periodontal diseases. The in vitro testing of the release showed that up to $80 \%$ of the drugs was released between 2 and 8 hours, keeping a very little residual rate up to 80 hours (Gad et al, 2017).

The results of the clinical study revealed a statistically significant improvement in PPD and Gingival index scores in the test group at 14 days and 1 month. Patients belonging to the test group showed greater reduction in PPD and gingival index scores when compared to patients of the control group at 14 days and 1 month. However there was no significant gain in clinical attachment levels within and between both groups. There was no significant reduction in probing pocket depths in the control group. None of the patients reported with any discomfort or pain after the placement of the gel in the periodontal pocket nor showed any signs of hypersensitivity to the prepared hydrogel. The lack of in-vitro evaluation of the mechanical and drug release properties of the gel, a small sample size and short duration of the clinical phase are the limitations of the current study.

\section{Conclusion:-}

Within the limits of the current study, it can be concluded that the prepared muco-adhesive hydrogel containing doxycycline and metronidazole showed potential for its use in treatment of Periodontitis as an adjunct to SRP. Local delivery of the hydrogel into the periodontal pocket led to significant reduction in probing pocket depths and gingival index scores. Further in-vitro studies evaluating its mechanical and biological properties and in-vivo studies with a larger sample size and longer duration are needed to establish its efficacy in treating Periodontitis.

\section{Acknowledgement:-}

The authors acknowledge Bapuji Pharmacy College, Davangere for providing assistance in the preparation of the hydrogel.

Table 1:-MIC Results

\begin{tabular}{|c|c|c|c|c|c|c|c|c|c|c|c|}
\hline $\begin{array}{l}\text { Sl. } \\
\text { No }\end{array}$ & Samples & $\begin{array}{l}100 \\
\mu \mathrm{l} / \mathrm{ml}\end{array}$ & $\begin{array}{l}50 \\
\mu \mathrm{l} / \mathrm{ml}\end{array}$ & $\begin{array}{l}25 \\
\mu 1 / m l\end{array}$ & $\begin{array}{l}12.5 \\
\mu 1 / \mathrm{ml}\end{array}$ & $\begin{array}{l}6.25 \\
\mu 1 / \mathrm{ml}\end{array}$ & $\begin{array}{l}3.12 \\
\mu 1 / \mathrm{ml}\end{array}$ & $\begin{array}{l}1.6 \\
\mu 1 / \mathrm{ml}\end{array}$ & $\begin{array}{l}0.8 \\
\mu 1 / \mathrm{ml}\end{array}$ & $\begin{array}{l}0.4 \\
\mu 1 / \mathrm{ml}\end{array}$ & $\begin{array}{l}0.2 \\
\mu \mathrm{l} / \mathrm{ml}\end{array}$ \\
\hline 1 & $\begin{array}{l}\text { P. } \\
\text { gingivalis }\end{array}$ & S & S & S & S & S & S & S & S & S & $\mathbf{R}$ \\
\hline 2 & $\begin{array}{l}\text { P. } \\
\text { intermedia }\end{array}$ & $S$ & $S$ & $S$ & $S$ & $S$ & $S$ & $S$ & $S$ & $S$ & $\mathbf{R}$ \\
\hline 3 & $\begin{array}{l}\text { F. } \\
\text { nucleatum }\end{array}$ & $S$ & $S$ & $S$ & $S$ & $S$ & $S$ & $S$ & $S$ & $S$ & $\mathbf{R}$ \\
\hline 4 & T. forsythia & $S$ & $S$ & $S$ & $S$ & $S$ & $S$ & $S$ & $S$ & $S$ & $\mathbf{R}$ \\
\hline
\end{tabular}

*Original table. S- Sensitive, R- Resistant

Table 2:-Intragroup comparison of PPD, CAL and GI in the control group

\begin{tabular}{|c|c|c|c|c|c|c|}
\hline $\begin{array}{l}\text { CONTROL } \\
\text { GROUP }\end{array}$ & \multirow{2}{*}{\multicolumn{2}{|c|}{$\begin{array}{l}\text { PPD } \\
(\text { Mean } \pm \text { SD })\end{array}$}} & \multirow{2}{*}{\multicolumn{2}{|c|}{$\begin{array}{l}\text { CAL } \\
(\text { Mean } \pm \text { SD) } \\
52+1.1 \mathrm{~mm}\end{array}$}} & \multicolumn{2}{|c|}{$\begin{array}{l}\text { GI } \\
(\text { Mean } \pm \text { SD })\end{array}$} \\
\hline BASELINE & & & & & $2.2 \pm 0.42$ & \\
\hline 14 DAYS & $5.3 \pm 0.94 \mathrm{~mm}$ & $\mathrm{p}=0.229$ & $4.9 \pm 0.99 \mathrm{~mm}$ & $\mathrm{p}=0.809$ & $1.4 \pm 0.84$ & $\mathrm{p}=0.001$ \\
\hline 1 MONTH & $5.3 \pm 0.67 \mathrm{~mm}$ & $\mathrm{p}=0.229$ & $4.9 \pm 1.1 \mathrm{~mm}$ & $\mathrm{p}=0.809$ & $1.0 \pm 0.47$ & \\
\hline
\end{tabular}

*Original table. PPD - Probing Pocket depth, CAL - Clinical Attachment loss, GI - Gingival Index, SD - Standard Deviation, $\mathrm{p}<0.05$ is statistically significant

Table 3:-Intragroup comparison of PPD, CAL and GI in the test group

\begin{tabular}{|c|c|c|c|c|c|c|}
\hline TEST GROUP & \multirow{2}{*}{\multicolumn{2}{|c|}{$\begin{array}{l}\text { PPD } \\
(\text { Mean } \pm \text { SD) } \\
5.7+0.67 \mathrm{~mm}\end{array}$}} & \multicolumn{2}{|l|}{$\begin{array}{l}\text { CAL } \\
(\text { Mean } \pm \text { SD })\end{array}$} & \multicolumn{2}{|c|}{$\begin{array}{l}\text { GI } \\
(\text { Mean } \pm \text { SD })\end{array}$} \\
\hline BASELINE & & & $5.0 \pm 0.81 \mathrm{~mm}$ & & $2.2 \pm 0.42$ & \\
\hline 14 DAYS & $4.5 \pm 0.84 \mathrm{~mm}$ & $p=0.009$ & $4.6 \pm 0.84 \mathrm{~mm}$ & $\mathrm{p}=0.539$ & $0.4 \pm 0.51$ & $\mathrm{p}=0.000$ \\
\hline 1 MONTH & $4.0 \pm 0.94 \mathrm{~mm}$ & $\mathrm{p}=0.000$ & $4.4 \pm 0.84 \mathrm{~mm}$ & $\mathrm{p}=0.260$ & $0.3 \pm 0.48$ & \\
\hline
\end{tabular}


*Original table. PPD - Probing Pocket depth, CAL - Clinical Attachment loss, GI - Gingival Index, SD - Standard Deviation, $\mathrm{p}<0.05$ is statistically significant

Table 4:-Intergroup comparison of PPD, CAL and GI at baseline and 1 month

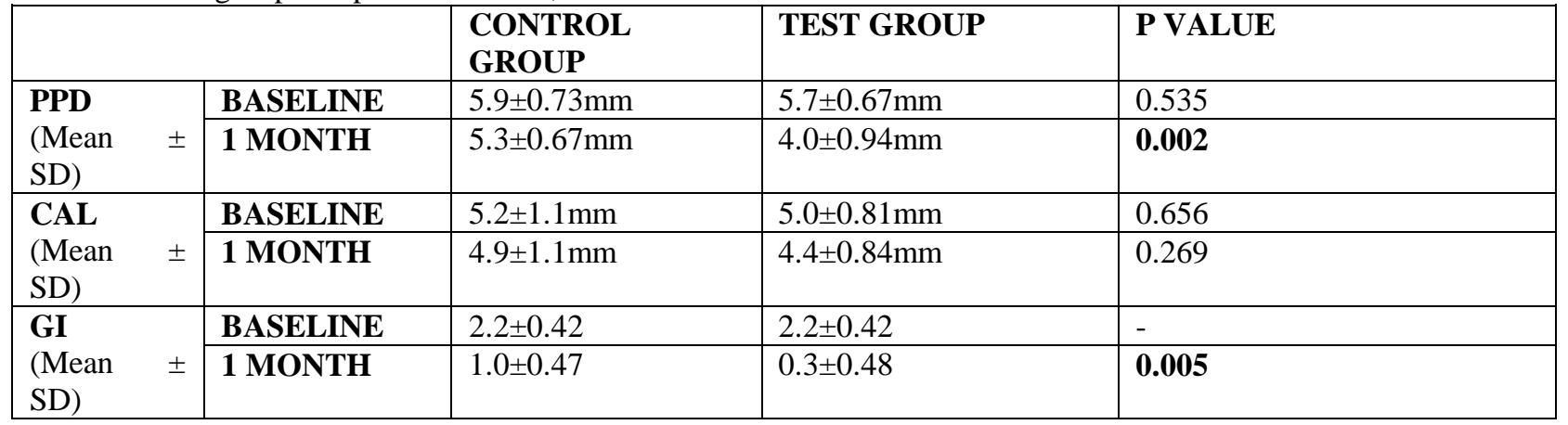

*Original table. PPD - Probing Pocket depth, CAL - Clinical Attachment loss, GI - Gingival Index, SD - Standard Deviation, $\mathrm{p}<0.05$ is statistically significant

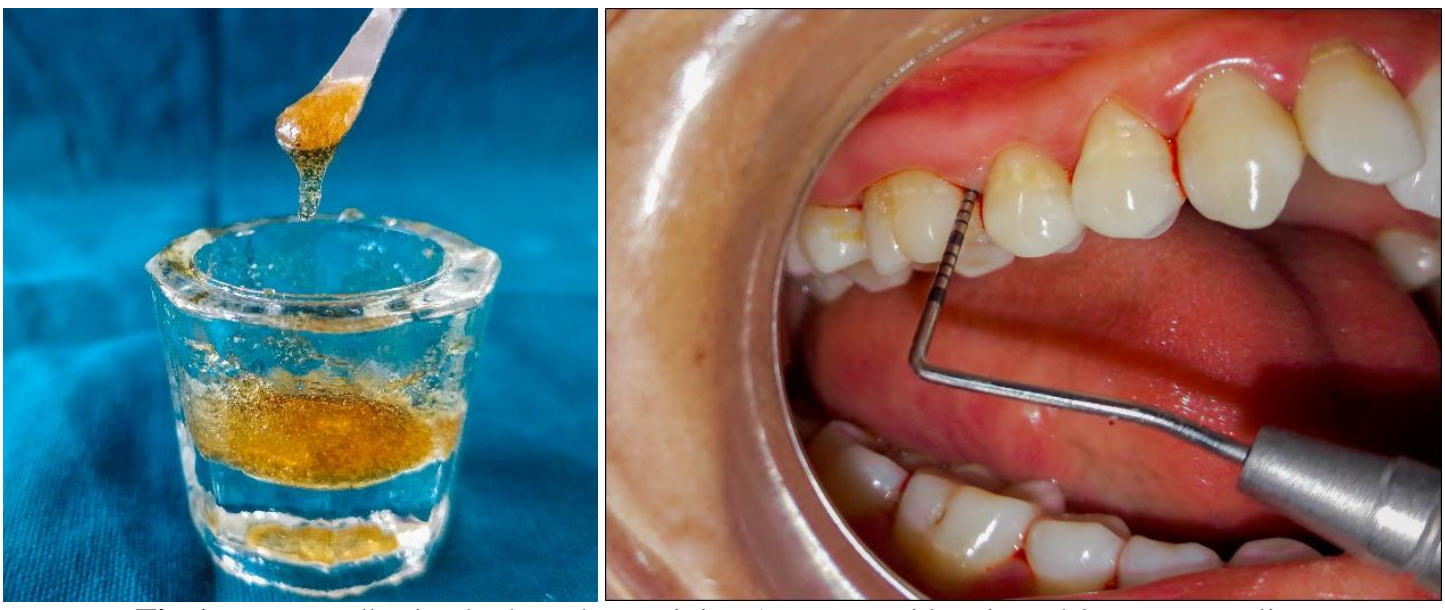

Fig 1: -Muco-adhesive hydrogel containing 1\% Metronidazole and 2\% Doxycycline

Fig 2:-Patients with Probing pocket depth of $5-7 \mathrm{~mm}$ were chosen for the study

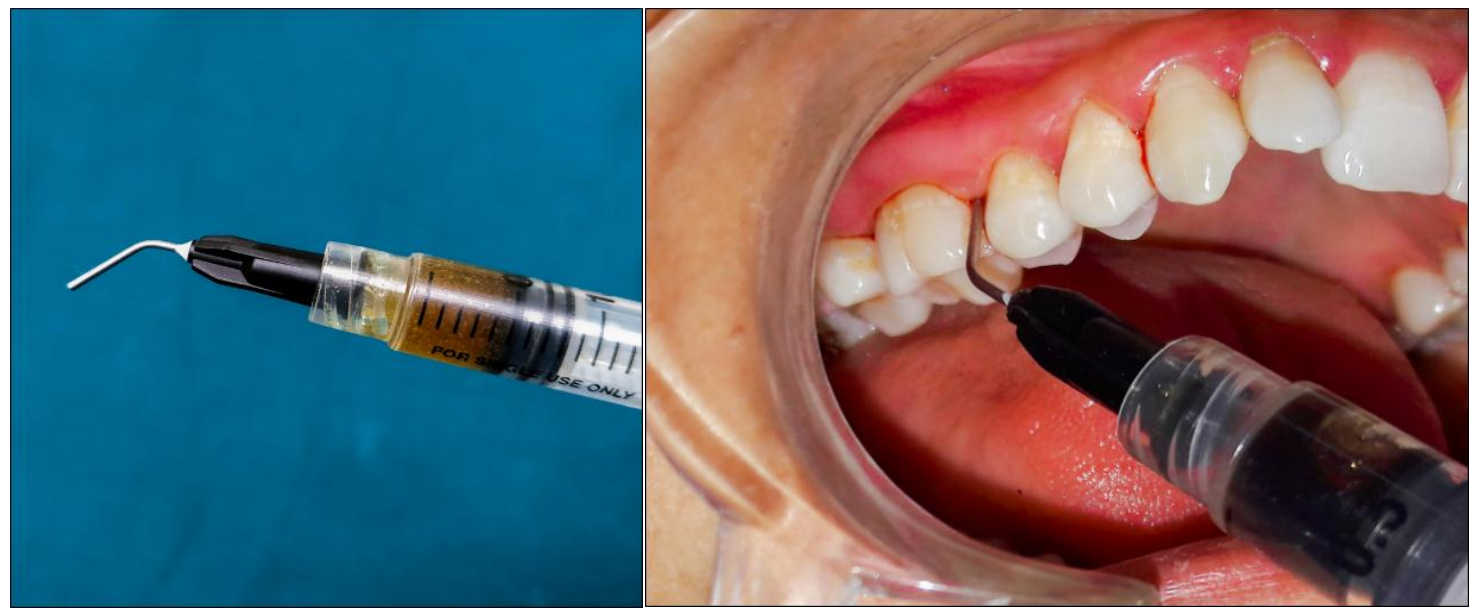

Fig 3a and Fig. 3b:-The gel was injected in to the periodontal pocket using a syringe with a wide-bore blunt needle. Approximately $0.5 \mathrm{ml}$ of gel was injected in the periodontal pocket around each tooth 


\section{References:-}

1. Chen, S. C., Wu, Y. C., Mi, F. L., Lin, Y. H., Yu, L. C., \& Sung, H. W. (2004). A novel pH-sensitive hydrogel composed of N, O-carboxymethyl chitosan and alginate cross-linked by genipin for protein drug delivery. Journal of Controlled Release, 96(2), 285-300.

2. de Abreu, F. R., \& Campana-Filho, S. P. (2009). Characteristics and properties of carboxymethylchitosan. Carbohydrate Polymers, 75(2), 214-221.

3. Gad, H. A., Kamel, A. O., Ezzat, O. M., El Dessouky, H. F., \& Sammour, O. A. (2017). Doxycycline hydrochloride-metronidazole solid lipid microparticles gels for treatment of periodontitis: development, in-vitro and in-vivo clinical evaluation. Expert opinion on drug delivery, 14(11), 1241-1251.

4. Golub, L. M., Lee, H. M., Ryan, M. E., Giannobile, W. V., Payne, J., \& Sorsa, T. (1998). Tetracyclines inhibit connective tissue breakdown by multiple non-antimicrobial mechanisms. Advances in dental research, 12(1), $12-26$.

5. Golub, L. M., Ramamurthy, N. S., McNamara, T. F., Greenwald, R. A., \& Rifkin, B. R. (1991). Tetracyclines inhibit connective tissue breakdown: new therapeutic implications for an old family of drugs. Critical Reviews in Oral Biology \& Medicine, 2(3), 297-321.

6. Golub, L. M., Suomalainen, K., \& Sorsa, T. (1992). Host modulation with tetracyclines and their chemically modified analogues. Current opinion in dentistry, 2, 80-90.

7. Greenstein, G. (2005). Changing periodontal concepts: treatment considerations. Compendium of continuing education in dentistry (Jamesburg, NJ: 1995), 26(2), 81-2.

8. Jepsen, S., Deschner, J., Braun, A., Schwarz, F., \& Eberhard, J. (2011). Calculus removal and the prevention of its formation. Periodontology 2000, 55(1), 167-188.

9. Löfmark, S., Edlund, C., \& Nord, C. E. (2010). Metronidazole is still the drug of choice for treatment of anaerobic infections. Clinical infectious diseases, 50(Supplement_1), S16-S23.

10. Sedlacek, M. J., \& Walker, C. (2007). Antibiotic resistance in an in vitro subgingival biofilm model. Oral microbiology and immunology, 22(5), 333-339.

11. Nastri, L., De Rosa, A., De Gregorio, V., Grassia, V., \& Donnarumma, G. (2019). A New Controlled-Release Material Containing Metronidazole and Doxycycline for the Treatment of Periodontal and Peri-Implant Diseases: Formulation and In Vitro Testing. International journal of dentistry, 2019.

12. Novak, M. J., Dawson, D. R., Magnusson, I., Karpinia, K., Polson, A., Polson, A., ... \& Powala, C. (2008). Combining host modulation and topical antimicrobial therapy in the management of moderate to severe periodontitis: a randomized multicenter trial. Journal of periodontology, 79(1), 33-41.

13. Papapanou, P. N., Sanz, M., Buduneli, N., Dietrich, T., Feres, M., Fine, D. H., ... \& Greenwell, H. (2018). Periodontitis: Consensus report of workgroup 2 of the 2017 World Workshop on the Classification of Periodontal and Peri-Implant Diseases and Conditions. Journal of periodontology, 89, S173-S182.

14. Preshaw, P. M. (2008). Host response modulation in periodontics. Periodontology 2000, 48(1), 92-110.

15. Larsen, T. (2002). Susceptibility of Porphyromonas gingivalis in biofilms to amoxicillin, doxycycline and metronidazole. Oral microbiology and immunology, 17(5), 267-271.

16. Tonetti, M. S., Greenwell, H., \& Kornman, K. S. (2018). Staging and grading of periodontitis: Framework and proposal of a new classification and case definition. Journal of clinical periodontology, 45, S149-S161.

17. Winkelhoff, A. J. V., Rams, T. E., \& Slots, J. (1996). Systemic antibiotic therapy in periodontics. Periodontology 2000, 10(1), 45-78. 